\title{
Fatores Influenciantes na Excelência de Cientistas Brasileiros
}

\author{
Maria Célia Bruno Mundim* \\ Pontifícia Universidade Católica de Campinas - PUC-Campinas, Campinas, SP, Brasil \\ ORCID: http://orcid.org/0000-0003-3450-7689 \\ Solange Muglia Wechsler** \\ Pontifícia Universidade Católica de Campinas - PUC-Campinas, Campinas, SP, Brasil \\ ORCID: http://orcid.org/0000-0002-9757-9113 \\ Leandro Silva Almeida*** \\ Universidade do Minho, Braga, Portugal \\ ORCID: http://orcid.org/0000-0002-0651-7014
}

\begin{abstract}
RESUMO
Este estudo objetivou investigar as variáveis pessoais e os fatores contextuais relacionados com a excelência de pesquisadores de ambos os gêneros e de diferentes áreas do conhecimento. A amostra foi constituída por 109 pesquisadores, bolsistas de produtividade do Conselho Nacional de Desenvolvimento Científico e Tecnológico (CNPq), que trabalham em instituições de ensino superior de várias regiões do Brasil. Utilizou-se o Questionário do Perfil de Excelência online formado por perguntas semi-abertas relacionadas a recursos pessoais, fatores acadêmicos e de fatores contextuais. Os resultados obtidos apontam para diferenças estatisticamente significantes de frequência de respostas entre os gêneros quanto à paixão pelo trabalho para enfrentar novos projetos de pesquisa, uso do suporte social, utilização de empregados para conciliar a vida pessoal com a profissional, na ocupação do tempo livre na realização de viagens e nas atividades com a família. Os resultados sugerem a natureza multivariada do fenômeno da excelência científica, convergindo na sua manifestação fatores pessoais e contextuais. Ambos os fatores merecem ser considerados quando se procura promover a sua realização profissional e produtividade.
\end{abstract}

Palavras-chave: excelência, investigação científica, traços da personalidade, fatores contextuais.

\section{Influential Factors in the Excellence of Brazilian Scientists}

\begin{abstract}
This study aimed to investigate personal variables and contextual factors related to the excellence of researchers of both genders and different areas of knowledge. The sample consisted of 109 researchers, National Scientific and Technological Development Council (CNPq) productivity fellows, who work in higher education institutions in various regions of Brazil. The Online Excellence Profile Questionnaire was used. This questionnaire consists of semi-open questions about personal resources, academic factors and contextual factors. The results obtained indicate statistically significant difference frequency of answers between
\end{abstract}


genders regarding the passion for work face new research projects, use of social support, use of employees to reconcile the personal life of the professional, in the occupation of free time with travel and in family activities. The results suggest a multivariate nature of the phenomenon of scientific excellence, converging in its manifestation personal and contextual factors. Both factors deserve to be considered when seeking to promote their professional fulfillment and productivity.

Keywords: excellence, scientific investigation, personality traits, contextual factors.

\section{Factores Influyentes en la Excelencia de los Científicos Brasileños}

\section{RESUMEN}

Este estudio tuvo como objetivo investigar las variables personales y los factores contextuales relacionados con la excelencia de los investigadores de ambos sexos y de diferentes áreas del conocimiento. La muestra consistió en 109 investigadores, becarios de productividad del Consejo Nacional de Desarrollo Científico y Tecnológico (CNPq), que trabajan en instituciones de educación superior en varias regiones de Brasil. Se utilizó el cuestionario de perfil de excelencia en línea. Este cuestionario consta de preguntas semiabiertas sobre recursos personales, factores académicos y factores contextuales. Los resultados obtenidos apuntan a diferencias estadísticamente significativas en la frecuencia de respuestas entre los géneros con respecto a la pasión por el trabajo para hacer frente a nuevos proyectos de investigación, el uso del apoyo social, el uso de empleados para conciliar la vida personal del profesional, la ocupación del tiempo libre para viajar y actividades familiares. Los resultados sugieren la naturaleza multivariante del fenómeno de la excelencia científica, convergiendo en su manifestación factores personales y contextuales. Ambos factores merecen ser considerados a la hora de promover su realización y productividad profesional.

Palabras clave: excelencia, investigación científica, rasgos de personalidad, factores contextuales.

O interesse da Psicologia pelo desenvolvimento potencial do indivíduo remonta desde meados do século passado (Hutz \& Pacico, 2016), ganhando o tema maior visibilidade acadêmica nas últimas décadas com os estudos sobre excelência acadêmica, desportiva e profissional no âmbito da Psicologia Positiva (Araújo, Cruz, \& Almeida, 2017; GarciaSantos, Almeida, \& Werlang, 2012; Seligman, 2002). A excelência, assim como o bem-estar, a felicidade, a qualidade de vida e a realização plena por meio de características positivas do indivíduo (a criatividade, a perseverança, a resiliência, a liderança, a sabedoria, etc.) são preconizados por esta abordagem psicológica recente (Seligman \& Csikszentmihalyi, 2000). 
Devido à sua complexidade e à diversidade de contextos de sua expressão, a excelência possui diferentes definições na literatura. Contudo, há uma compreensão comum entre os estudiosos de que ela refere-se à capacidade ou desempenho superior de um indivíduo quando comparado com aqueles do mesmo campo de atuação (Trost, 2000). Para o alcance da excelência ou para sua manutenção durante algum período de tempo, no entanto, múltiplas são as variáveis intervenientes. Uma das linhas de maior produtividade científica na área, por exemplo, propõe que a aquisição de conhecimento somada à prática intencional prolongada de uma pessoa em dada área, seja essencial para se atingir e manter a excelência (Ericsson, Hoffman, Kozbelt, \& Williams, 2018).

Nas Ciências, a excelência é avaliada por meio da produção de conhecimento que envolve status na carreira dos pesquisadores e volume de financiamento de projetos (González Ramos \& Benavente, 2017). Tomando critérios das agências de financiamento e da qualidade das instituições de pesquisa, a excelência científica associa-se à qualidade e à quantidade de publicações em revistas de alto impacto, ao trabalho em colaboração nas redes científicas e com pesquisadores estrangeiros, à obtenção de premiações na área de atuação, ter citações expressivas na área de conhecimento e/ou ter projetos financiados (Tahamtan, Afshar, \& Ahamdzadeh, 2016; Zanotto, 2006; Zeng et al., 2017). Assim, Van den Brink e Benschop (2012) sintetizam a excelência acadêmica como decorrente da combinação bemsucedida dos fatores - competências profissionais, habilidades individuais e conexões de rede.

Quanto aos aspectos que são determinantes ao alcance da excelência, vários autores julgam os de caráter ambiental e os de cunho pessoal (Simonton, 1999; Wai, Lubinski, \& Benbow, 2005; Ziegler \& Phillipson, 2012). Desse modo, variáveis como a personalidade, a cognição e a motivação são consideradas tão importantes quanto a oportunidade, a influência de mentores, o contexto familiar e o profissional (Almeida \& Wechsler, 2015; Csikszentmihalyi, 1997; Fleith, 2007; Pfeiffer, 2015).

Poucos são os estudos internacionais que descrevem o cientista eminente. Dentre esses estudos, está o realizado por Tang e Kaufman (2017) que constatou os seguintes adjetivos que o caracteriza: curioso, auto-confiante, íntegro, intuitivo, dotado de pensamento divergente e motivado. Especificamente sobre a motivação dos pesquisadores, Lam (2011) refere que os diferentes graus de motivos intrínsecos (como a satisfação) e extrínsecos (reconhecimento, recompensas financeiras e de carreira) podem ocorrer em função do grau de congruência entre os valores pessoais e aqueles subjacentes à atividade científica. A excelência apenas se atinge com profissionais entusiasmados e totalmente imersos em seu trabalho (Tauhed, Rasdi, Ibrahim, \& Samah, 2019), podendo a motivação intrínseca mediar a relação entre as 
capacidades e os seus níveis elevados de desempenho no trabalho (Ringelhan, Wollersheim, Welpe, Fiedler, \& Spörrle, 2013). Ainda no que diz respeito à motivação intrínseca dos cientistas, a intuição e, sobretudo, a paixão pelo trabalho que sentem são as principais causas que os movem, de acordo com Grinnell (2009).

Entretanto, para que os recursos pessoais, como a motivação, se expressem em excelência, importam contextos sociais e institucionais favoráveis (Mundim, Wechsler, \& Almeida, 2019). Ambientes de trabalho pautados por inovação, segurança nas relações interpessoais, incentivos à produtividade, conciliação de papeis e abertura à experiência são decisivos (Christensen, Dyrstad, \& Innstrand, 2018). Na área da pesquisa, por exemplo, as políticas editoriais de revistas e de instituições podem ser determinantes na quantidade e qualidade dos artigos publicados (Castro \& Santo, 2017; De Meis, Velloso, Lannes, Carmo, \& De Meis, 2003).

Há estudos que analisam como os cientistas criam e alimentam as suas carreiras profissionais de excelência. A conciliação de papéis de trabalho, família e vida social aparece destacada na superação de desafios, apontando a utilização de estratégias tais como o gerenciamento do tempo, o engajamento com as atividades e o networking diários (Luthar, 2017; Tauhed, Rasdi, Ibrahim, \& Samah, 2019), podendo as mulheres apresentar maiores dificuldades nessa conciliação pelo maior volume de responsabilidades atribuídas, sobretudo em termos familiares (Dubbelt, Rispens, \& Demerouti, 2016; Ecklund \& Lincoln, 2016). Logo, a excelência nesta área profissional implica altos níveis de envolvimento no trabalho (Retzbach, Otto, \& Maier, 2016), reconhecendo a importância da sua criatividade, por meio de analogias, imaginação e simulação mental, na colocação de novos problemas nas áreas de conhecimento e respectiva solução (Clement, 2008; DeHaan, 2011; Hadzigeorgiou, 2016).

A excelência das mulheres ou excelência no feminino tem sido objeto de particular interesse na academia. Além da sobrecarga de funções no ambiente doméstico, cientistas do gênero feminino podem sair prejudicadas pelos períodos de não-publicação devido à licençamaternidade e aos cuidados com filhos pequenos (Nielsen, 2016; Sewell \& Barnett, 2019). No mesmo sentido, o estudo de Damaske, Ecklund, Lincoln e White (2014) sugere que cientistas do sexo masculino que têm buscado relacionamentos mais igualitários no casamento, compartilhando as atividades domésticas e a educação dos filhos, estão tendendo a vivenciar mais o conflito entre a paternidade e as tarefas científicas. A conciliação das demandas da família e da profissão, sobretudo quando a maior parte do tempo é despendida com o trabalho, como ocorre com as pesquisadoras eminentes, justifica o recurso por estas profissionais do apoio social e familiar (Prado \& Fleith, 2012). As cientistas do gênero feminino recorrem a 
outros membros da família ou a empregadas domésticas, conforme Melo, Lastres e Marques (2004). Em entrevista com professoras pesquisadoras de uma universidade pública no Brasil, Montagner e Montagner (2010), por exemplo, observaram que algumas delas valem-se do auxílio de mães, sogras, babás e/ou empregadas domésticas para manterem elevada a sua produção científica.

Além do suporte familiar e profissional, o lazer se faz importante para preservação da saúde mental e física dos cientistas. Todavia, a diversidade das atividades acadêmicas sobrecarregam os docentes pesquisadores, invadindo, assim, seu cotidiano particular e inviabilizando o tempo para o descanso, o lazer, o convívio familiar e social (Borsoi \& Pereira, 2013; Gómez, 2015). Pesquisadores de instituições públicas brasileiras referem trabalhar de 18,6 a 30 horas semanais em casa no período noturno, nos fins de semana e em feriados para concluir tarefas pendentes. Eles também pontuam que procuram organizar sua jornada de trabalho de modo racional para dividir o tempo disponível entre as atividades de docência, pesquisa, encargos administrativos, elaboração de trabalhos científicos, viagens para participação em congressos ou bancas e assim por diante (Borsoi, 2012).

Também em termos de aproveitamento dos tempos livres se observa uma diferença segundo o gênero dos cientistas envolvidos. Mattingly e Sayer (2006) perceberam que devido às pressões psicológicas que o gênero feminino sofre para cuidar das tarefas da casa, o lazer acaba por ter menos efeitos positivos sobre a saúde delas. Isto, porque as atividades de lazer individual, familiar e/ou com amigos, variam conforme a presença de filhos pequenos e da estrutura de apoio que as mulheres dispõem com empregados e familiares (Jablonski, 2010).

Diante do exposto, este estudo teve como objetivo avaliar as variáveis pessoais e contextuais que influenciam e caraterizam a excelência de pesquisadores brasileiros, de acordo com o gênero e as áreas do conhecimento. Em particular, serão analisadas suas motivações, suas atitudes diante de pressões e problemas relacionados ao trabalho científico, incluindo as atividades realizadas nos tempos livres.

\section{Método}

\section{Participantes}

Participaram deste estudo 109 pesquisadores bolsistas de produtividade do Conselho Nacional de Desenvolvimento Científico e Tecnológico (CNPq) 1A (33,9\%), 1B (36,6\%), 1C $(23,8 \%)$ e $1 \mathrm{D}(5,5 \%)$ advindos de instituições públicas em sua maioria $(84,4 \%)$. A idade 
média dos participantes foi de 58,6 anos (desvio-padrão de 8,92), sendo que a idade mínima foi de 36 anos e a máxima de 81 anos, com predomínio do estado civil de casado/união estável (83,5\%). Quanto ao gênero e às áreas científicas, 29 participantes eram das Biológicas (26,6\%) com 34\% de mulheres, 28 das Humanas (25,7\%) com 53\% de mulheres, 27 da Saúde $(24,8 \%)$ com $51 \%$ de mulheres e 25 das Exatas/Tecnologias $(22,9 \%)$ com $44 \%$ de mulheres.

\section{Instrumento}

Utilizou-se o Questionário do Perfil de Excelência, desenvolvido pelos autores e constituído por sete questões semi-abertas relacionadas a recursos pessoais (para lidar com imprevistos e com novos projetos), fatores acadêmicos (procedimento de trabalho, atitudes diante de pressões por produtividade, aspectos institucionais de ensino superior que propiciam a excelência científica) e de fatores contextuais (atividades de lazer, maneira de conciliar a esfera profissional com a pessoal). O questionário foi concebido para uma versão online pelos autores que se basearam na literatura referente aos fatores que ocasionam a excelência profissional. Na página inicial havia o termo de consentimento informado e, após aceitação do participante, o mesmo prosseguia para as questões a serem respondidas em uma nova aba.

\section{Procedimentos}

Depois do estudo ser aprovado pelo Comitê de Ética em Pesquisa com Seres Humanos sob o parecer $\mathrm{n}^{\circ}$ 51199415.1.0000.5481, foi realizada busca para aquisição de informações dos possíveis participantes através da Plataforma Lattes. O critério de pesquisadores bolsistas de produtividade do CNPq 1A, 1B, 1C e 1D das áreas de Saúde, Biológicas, Humanas e Exatas/Tecnologias foi utilizado nessa busca. Os pesquisadores identificados foram contatados via correio eletrônico por meio da plataforma Survey Monkey, com o intuito de expor o objetivo do estudo e convidá-los a participar do mesmo. Posteriormente, realizou-se a análise do conteúdo das respostas e recorreu-se ao teste Qui-quadrado para testar diferenças nas frequências de respostas entre subgrupos. 


\section{Resultados}

Na Tabela 1 são apresentados os resultados dos participantes sobre a questão referente aos recursos ou competências pessoais que utilizam para enfrentarem problemas ou imprevistos que possam surgir no decurso dos seus projetos de pesquisa. Algumas respostas foram agrupadas para facilitar esta análise. Assim, juntaram-se os participantes que responderam recorrer à criatividade ou improvisação, assim como aqueles que responderam recorrer ao sentido crítico ou planejamento da ação.

\section{Tabela 1}

Recursos para lidar com imprevistos na execução de seus projetos científicos

\begin{tabular}{lccccccc}
\hline Recursos & Global & Feminino & Masculino & Biológicas & Exatas & Humanas & Saúde \\
\hline & $f(\%)$ & $f(\%)$ & $f(\%)$ & $f(\%)$ & $f(\%)$ & $f(\%)$ & $f(\%)$ \\
\hline $\begin{array}{l}\text { Criatividade/ } \\
\text { Improvisa }\end{array}$ & $76(69,7 \%)$ & $44(74,6 \%)$ & $32(64,0 \%)$ & $21(72,4 \%)$ & $20(80,0 \%)$ & $20(71,4 \%)$ & $15(55,6 \%)$ \\
Objetividade & $56(51,4 \%)$ & $28(47,5 \%)$ & $28(56,0 \%)$ & $17(58,6 \%)$ & $13(52,0 \%)$ & $11(39,3 \%)$ & $15(55,6 \%)$ \\
Senso & & & & & & & \\
critico/Planeja & $83(76,1 \%)$ & $42(71,2 \%)$ & $41(82,0 \%)$ & $21(72,4 \%)$ & $18(72,0 \%)$ & $21(75,0 \%)$ & $23(85,2 \%)$ \\
\hline
\end{tabular}

Os resultados sugerem que predomina na amostra a estratégia de usar o sentido crítico e planejar as ações $(76,1 \%)$ em resposta aos problemas e improvisos que surjam, seguida pelo recurso à criatividade e/ou improvisação (69,7\%). A análise objetiva das situações surge apontada por cerca de $50 \%$ dos participantes, sendo esta estratégia mais seguida pelos participantes do sexo feminino, enquanto o recurso ao senso crítico e planejamento da ação surge mais mencionado pelos participantes do sexo masculino (82,0\%). A criatividade e/ou improvisação aparecem com taxa mais elevada na área científica de Exatas (80,0\%), a análise objetiva da situação é mais frequente na área Biológica $(58,6 \%)$ e o sentido crítico e/ou planejamento da ação aparecem mais elevados na área de Saúde (85,2\%). As discrepâncias assinaladas nas percentagens de respostas tomando o gênero e a área científica dos participantes, no entanto, não se apresentam estatisticamente significantes.

$\mathrm{Na}$ Tabela 2 foram elencadas as respostas dos participantes sobre os sentimentos e motivações que lhes suscitam enfrentar novos projetos de pesquisa. As respostas foram distribuídas por curiosidade, possibilidades de desenvolvimento pessoal, aperfeiçoamento 
contínuo, paixão pela atividade e reconhecimento (aqui integra-se reconhecimento social, reconhecimento financeiro e resposta a demandas externas).

Tabela 2

Fatores de motivação frente a um novo projeto

\begin{tabular}{|c|c|c|c|c|c|c|c|}
\hline $\begin{array}{l}\text { Geradores de } \\
\text { motivação }\end{array}$ & Global & Feminino & Masculino & Biológicas & Exatas & Humanas & Saúde \\
\hline & $f(\%)$ & $f(\%)$ & $f(\%)$ & $f(\%)$ & $f(\%)$ & $f(\%)$ & $f(\%)$ \\
\hline Curiosidade & $71(23,8 \%)$ & $30(21,2 \%)$ & $41(26,2 \%)$ & $21(26,5 \%)$ & $17(25,3 \%)$ & $16(21,6 \%)$ & $17(22,0 \%)$ \\
\hline $\begin{array}{l}\text { Desenvolvimento } \\
\text { potencial }\end{array}$ & $46(15,4 \%)$ & $23(16,3 \%)$ & $22(14,1 \%)$ & $14(17,7 \%)$ & $9(13,4 \%)$ & $10(13,5 \%)$ & $12(15,5 \%)$ \\
\hline $\begin{array}{l}\text { Aperfeiçoamento } \\
\text { contínuo }\end{array}$ & $62(20.8 \%)$ & $32(22,6 \%)$ & $30(19.2 \%)$ & $17(21,5 \%)$ & $12(17.9 \%)$ & $14(18,9 \%)$ & $19(24,6 \%)$ \\
\hline $\begin{array}{l}\text { Paixão pelo que } \\
\text { faz }\end{array}$ & $85(28,5 \%)$ & $43(30,4 \%)$ & $42(26,9 \%)$ & $24(30,3 \%)$ & $21(31,3 \%)$ & $21(28,3 \%)$ & $19(24,6 \%)$ \\
\hline Reconhecimento & $34(11,4 \%)$ & $13(9,2 \%)$ & $21(13,4 \%)$ & $3(3,7 \%)$ & $8(11,9 \%)$ & $13(17,5 \%)$ & $10(12,9 \%)$ \\
\hline
\end{tabular}

Os resultados mostram uma relativa dispersão das respostas, havendo ligeira superioridade na frequência de respostas mencionando a paixão pelo que fazem $(28,5 \%)$, na qual foi verificada diferença significativa $\left(\chi^{2}=3,46, \mathrm{gl}=1, p=0,05\right)$ a favor das mulheres. curiosidade e o aperfeiçoamento contínuo também foram bastante referenciados (entre 20 e $30 \%$ ) nos vários subgrupos da amostra, considerando o gênero e área científica de atuação, embora sem diferenças com significado estatístico para ambas variáveis.

Na Tabela 3 os participantes assinalaram as suas formas de resposta ou formas de enfrentamento face à pressão por maior produtividade na sua atividade. As respostas repartem-se por maior motivação, fixar prioridades, busca de suporte (social, familiar), pedido de apoio (profissional, religioso) e relaxamento. 


\section{Tabela 3}

Reação frente às pressões por maior produtividade no trabalho

\begin{tabular}{|c|c|c|c|c|c|c|c|}
\hline $\begin{array}{l}\text { Reação às } \\
\text { pressões }\end{array}$ & Global & Feminino & Masculino & Biológicas & Exatas & Humanas & Saúde \\
\hline & $f(\%)$ & $f(\%)$ & $f(\%)$ & $f(\%)$ & $f(\%)$ & $f(\%)$ & $f(\%)$ \\
\hline Motivação & $27(11,2 \%)$ & $10(8,0 \%)$ & $17(14,7 \%)$ & $7(12.2 \%)$ & $9(17,6 \%)$ & $6(9,0 \%)$ & $5(7,5 \%)$ \\
\hline $\begin{array}{l}\text { Flexibilização } \\
\text { de horários }\end{array}$ & $38(15,8 \%)$ & $20(16,0 \%)$ & $18(15,6 \%)$ & $10(17,5 \%)$ & $8(15,6 \%)$ & $12(18,1 \%)$ & $8(12,1 \%)$ \\
\hline Prioriza & $93(38,7 \%)$ & $43(34,4 \%)$ & $50(43,4 \%)$ & $25(43,8 \%)$ & $19(37,2 \%)$ & $24(36,3 \%)$ & $25(37,8 \%)$ \\
\hline Suporte & & & & & & & \\
\hline $\begin{array}{l}\text { social/ } \\
\text { familiar }\end{array}$ & $26(10,8 \%)$ & $19(15,2 \%)$ & $7(6,0 \%)$ & $2(3,5 \%)$ & $6(11,7 \%)$ & $10(15,1 \%)$ & $8(12,1 \%)$ \\
\hline $\begin{array}{l}\text { Apoio } \\
\text { profissional }\end{array}$ & $20(8,3 \%)$ & $14(11,2 \%)$ & $6(5,2 \%)$ & $5(8,7 \%)$ & $4(7,8 \%)$ & $6(9,0 \%)$ & $5(7,5 \%)$ \\
\hline Relaxamento & $36(15 \%)$ & $19(15,2 \%)$ & $17(14,7 \%)$ & $8(14,0 \%)$ & $5(9,8 \%)$ & $8(12,1 \%)$ & $15(22,7 \%)$ \\
\hline
\end{tabular}

Os resultados mostram que um maior número de participantes $(38,7 \%)$ faz planejamento e escolhas, priorizando a sua atividade de pesquisa. Esta tendência ocorre nos dois gêneros e nas quatro áreas científicas de atuação (ligeiramente inferior nos participantes do gênero feminino com $34,4 \%$ de respondentes). Segue-se o recurso à flexibilização de horários e ao relaxamento, contudo com percentagens respectivas de ocorrência bastante flutuante em função da área científica (por exemplo, maior flexibilização de horários que relaxamento nas Exatas versus maior relaxamento que flexibilização de horários na Saúde). Entretanto, foram observadas diferenças estatísticas segundo o gênero no suporte social $\left(\chi^{2}=\right.$ $7,31, \mathrm{gl}=1, p=0,007)$ e no apoio profissional $\left(\chi^{2}=5,28, \mathrm{gl}=1, p=0,022\right)$, em ambos os casos favoráveis às mulheres. Quanto às áreas científicas, também notou-se diferença significativa no suporte social, justificada pela maior referenciação na área das Humanas $\left(\chi^{2}=\right.$ $9,65, \mathrm{gl}=3, p=0,022)$ e na dimensão relaxamento, decorrente da maior referenciação na área da Saúde $\left(\chi^{2}=8,75, \mathrm{gl}=3, p=0,033\right)$.

$\mathrm{Na}$ Tabela 4, os participantes expressaram o modo como conciliam ou não a esfera pessoal com a profissional. Dos 109 respondentes, 4 referiram que não conseguiam fazer essa conciliação, sendo que se repartem pelas várias áreas científicas, embora mais concentrados no sexo feminino (três dos quatro respondentes). 


\section{Tabela 4}

Procedimento de conciliação da vida profissional e pessoal

\begin{tabular}{|c|c|c|c|c|c|c|c|}
\hline $\begin{array}{l}\text { Concilia vida } \\
\text { pessoal e } \\
\text { profissional }\end{array}$ & Global & Feminino & Masculino & Biológicas & Exatas & Humanas & Saúde \\
\hline & $f(\%)$ & $f(\%)$ & $f(\%)$ & $f(\%)$ & $f(\%)$ & $f(\%)$ & $f(\%)$ \\
\hline $\begin{array}{l}\text { Com ajuda da } \\
\text { familia }\end{array}$ & $85(78,0 \%)$ & $48(81,4 \%)$ & $37(74,0 \%)$ & $23(79,3 \%)$ & $18(72,0 \%)$ & $23(82,1 \%)$ & $21(77,8 \%)$ \\
\hline $\begin{array}{l}\text { Com ajuda de } \\
\text { colegas/amigos }\end{array}$ & $4541,3 \%)$ & $23(39,0 \%)$ & $22(44,0 \%)$ & $14(48,3 \%)$ & $7(28,0 \%)$ & $13(46,4 \%)$ & $11(40,7 \%)$ \\
\hline Sozinho/religião & $23(21,1 \%)$ & $14(23,7 \%)$ & $9(18,0 \%)$ & $7(24,1 \%)$ & $5(20,0 \%)$ & $4(14,3 \%)$ & $7(25.6 \%)$ \\
\hline $\begin{array}{l}\text { Com ajuda de } \\
\text { empregados }\end{array}$ & $31(28,4 \%)$ & $6(10,2 \%)$ & $25(50,0 \%)$ & $9(31,0 \%)$ & $4(16,0 \%)$ & $10(35,7 \%)$ & $8(29,6 \%)$ \\
\hline
\end{tabular}

Referente aos recursos e à forma como os respondentes procuram conciliar a sua vida profissional e pessoal, verifica-se que a grande maioria recorre à ajuda da família, situação esta que abrange entre 70 e $80 \%$ dos respondentes sejam nos dois gêneros, seja nas quatro áreas científicas. Por esta razão, e mesmo havendo um ligeiro aumento dessa percentagem junto dos respondentes do sexo feminino e nos profissionais da área das Humanas, tal discrepância não se apresenta estatisticamente significante. $\mathrm{O}$ recurso à ajuda de empregados ou colaboradores é maior no sexo masculino, atingindo metade dos homens, contra apenas $10 \%$ no sexo feminino. Neste caso a diferença nos efetivos atinge significado estatístico $\left(\chi^{2}=\right.$ 21,097, $\mathrm{gl}=1, p<0,001$ ), a favor do sexo masculino. Registra-se, ainda, uma diversidade de efetivos que recorrem à ajuda dos empregados pelas quatro áreas científicas, oscilando os valores percentuais entre $16 \%$ no caso das Exatas e 36\% nas Humanas. Apesar destas discrepâncias, o valor do qui-quadrado calculado tomando os quatro agrupamentos não se apresenta estatisticamente significante. Nos demais recursos utilizados para a conciliação da vida pessoal com a profissional também não foram encontradas diferenças significativas.

Por fim, na Tabela 5, são apresentados os resultados que descrevem como os participantes do estudo ocupam o seu tempo livre ou momentos de lazer. 


\section{Tabela 5}

Atividades nos momentos de lazer/tempo livre

\begin{tabular}{|c|c|c|c|c|c|c|c|}
\hline $\begin{array}{l}\text { Tempo } \\
\text { Livre }\end{array}$ & Global & Feminino & Masculino & Biológicas & Exatas & Humanas & Saúde \\
\hline & $f(\%)$ & $f(\%)$ & $f(\%)$ & $f(\%)$ & $f(\%)$ & $f(\%)$ & $f(\%)$ \\
\hline $\begin{array}{l}\text { Atividades } \\
\text { Fisicas }\end{array}$ & $71(15,0 \%)$ & $35(14,7 \%)$ & $36(15,3 \%)$ & $17(13,9 \%)$ & $13(12,2 \%)$ & $19(16,1 \%)$ & $22(17,5 \%)$ \\
\hline $\begin{array}{l}\text { Atividades } \\
\text { Artisticas/ }\end{array}$ & $85(18,0 \%)$ & $42(17,7 \%)$ & $43(18,3 \%)$ & $21(17,2 \%)$ & $19(17,9 \%)$ & $23(19,4 \%)$ & $22(17,5 \%)$ \\
\hline Culturais & & & & & & & \\
\hline $\begin{array}{l}\text { Atividades } \\
\text { Intelectuais/ } \\
\text { Profissionais }\end{array}$ & $107(22,7 \%)$ & $54(22,7 \%)$ & $53(22,6 \%)$ & $28(22,9 \%)$ & $25(23,5 \%)$ & $27(22,8 \%)$ & $27(21,6 \%)$ \\
\hline Viagens & $81(17,1 \%)$ & $43(18,1 \%)$ & $38(16,2 \%)$ & $19(15,5 \%)$ & $20(18,8 \%)$ & $21(17,7 \%)$ & $21(16,8 \%)$ \\
\hline $\begin{array}{l}\text { Passa com } \\
\text { Familia }\end{array}$ & $86(18,2 \%)$ & $44(18,5 \%)$ & $42(17.9 \%)$ & $24(19,6 \%)$ & $20(18,8 \%)$ & $20(16,9 \%)$ & $22(17,5 \%)$ \\
\hline $\begin{array}{l}\text { Passa com } \\
\text { Amigos }\end{array}$ & $41(8,7 \%)$ & $19(8,0 \%)$ & $22(9.4 \%)$ & $13(10,6 \%)$ & $9(8,4 \%)$ & $8(6,7 \%)$ & $11(8.7 \%)$ \\
\hline
\end{tabular}

Os resultados obtidos apontam para uma grande diversidade de atividades ou formas de ocupar o tempo livre. Apesar das oscilações nas percentagens obtidas, em nenhuma das situações se observa uma diferença significativa tomando as quatro áreas científicas. Contudo, olhando a variável gênero, foram encontradas diferenças significantes a favor dos homens na realização de viagens $\left(\chi^{2}=6,61, \mathrm{gl}=1, p=0,010\right)$ e quase significativa nas atividades artísticas/culturais $\left(\chi^{2}=3,14, \mathrm{gl}=1, p=0,08\right)$, ao mesmo tempo que se verificam a favor das mulheres diferenças estatisticamente significativas nas atividades com a família $\left(\chi^{2}=4,60\right.$, gl $=1, p=0,032)$ e uma diferença quase significativa nas atividades intelectuais/profissionais $\left(\chi^{2}\right.$ $=3,44, \mathrm{gl}=1, p=0,06)$. 


\section{Discussão e Conclusão}

O intuito deste estudo foi o de verificar os fatores pessoais e contextuais que favorecem a excelência de mulheres e homens que atuam como pesquisadores em diferentes áreas do conhecimento. Os resultados indicaram não haver diferença significativa entre os gêneros nem entre as áreas científicas quanto aos recursos ou competências pessoais que utilizam para enfrentarem problemas ou imprevistos que possam aparecer no decurso dos seus projetos de pesquisa. Apesar desses resultados, houve predominância da amostra em utilizar o sentido crítico e planejamento das ações em resposta aos problemas e improvisos que surjam, seguida pelo recurso da criatividade e/ou da improvisação. Esses dados vem, em parte, ao encontro com a literatura, já que para Clement (2008), DeHaan (2011) e Hadzigeorgiou (2016) os cientistas utilizam muito da criatividade, seja por meio de analogias, imaginação ou simulação mental na solução de problemas, assim como o engajamento com o trabalho frente as incertezas e problemas científicos (Retzbach et al., 2016).

Sobre os sentimentos e motivações frente a novos projetos de pesquisa, os resultados mostram uma relativa dispersão das respostas dos participantes. Há, entretanto, ligeira superioridade na frequência de respostas mencionando a paixão pelo que fazem a favor das mulheres, sendo este aliás um fator presente em diversas pesquisas sobre os determinantes ou variáveis associadas ao atingir e manter performances excelentes nas mais diversas áreas. Grinnell (2009) também refere a paixão pelo trabalho como principal causa de motivação entre os cientistas. A curiosidade e o aperfeiçoamento contínuo são fontes de motivação também bastante referenciadas nos vários subgrupos da amostra quando o gênero e área científica de atuação são considerados, já que a motivação intrínseca interpõe-se entre a satisfação que sentem e o desempenho no trabalho (Ringelhan et al., 2013).

Quanto ao modo como reagem frente às pressões por maior produtividade no trabalho, a amostra apresentou diferença significativa no que refere ao uso do suporte social e apoio profissional a favor das mulheres. Também observou-se diferença significativa na utilização do suporte social para a área de Humanas, assim como do emprego de relaxamento mais mencionado pelos profissionais da área da saúde. Devido à ocorrência de maior percentagem de mulheres que atuam na área científica das Humanas, esta maior frequência de recurso ao suporte junto de colegas e da família pode estar associado à maior sobrecarga de tarefas que as mulheres apresentam, sendo apontado na literatura sobre a excelência profissional a necessidade das mulheres conseguirem melhor gerir o tempo e conciliar a sua carreira profissional com outras responsabilidades em termos familiares e sociais (equilibrar vários 
papéis). Conforme Luthar (2017), Tauhed, Rasdi, Ibrahim e Samah (2019), a conciliação de papeis de trabalho, família e vida social aparece destacada na superação de desafios dos cientistas, apontando a utilização de estratégias tais como o gerenciamento do tempo, o engajamento com as atividades e o networking. Entretanto, as mulheres podem apresentar maiores dificuldades nessa conciliação pelo maior volume de responsabilidades atribuídas, principalmente em termos familiares (Dubbelt et al., 2016; Ecklund \& Lincoln, 2016). Além do uso de estratégias, os profissionais das Ciências buscam organizar sua rotina de trabalho de modo mais objetivo a fim de dar conta das diversas tarefas - docência, pesquisa, encargos administrativos, elaboração de trabalhos científicos, viagens para participação em congressos ou bancas, dentre outras (Borsoi, 2012).

Com relação ao modo como conciliam ou não a esfera pessoal com a profissional, notou-se que a grande maioria recorre à ajuda da família, situação esta que abrange os dois gêneros e as quatro áreas científicas. Os homens demonstraram também contar mais com a ajuda de empregados ou colaboradores do que as mulheres, havendo significância estatística a favor deles neste quesito. Isto não está de acordo com o que tem sido identificado por estudiosos do tema, pois são os cientistas do sexo feminino e não os homens que buscam mais ajuda familiar e/ou de empregadas domésticas (Melo et al., 2004). Em estudo de Montagner e Montagner (2010), por exemplo, observou-se que algumas cientistas valem-se do auxílio de mães, sogras, babás e/ou empregadas domésticas. Prado e Fleith (2012) também verificaram a utilização de apoio social e familiar por parte de pesquisadoras eminentes.

A respeito do uso do tempo livre ou momentos de lazer pelos participantes, os resultados obtidos apontam para uma grande diversidade de atividades e formas de ocupar o tempo livre em ambos gêneros e áreas científicas. Considerando a variável gênero, encontramos diferenças significativas a favor dos homens na realização de viagens e quase significativa nas atividades artísticas/culturais, ao mesmo tempo que se verificam a favor das mulheres diferenças significativas nas atividades com a família e uma diferença quase significativa nas atividades intelectuais/profissionais. Para Borsoi e Pereira (2013) e Gómez (2015), o lazer, o descanso, o convívio familiar e social dos pesquisadores são comprometidos pela sobrecarga de trabalho que possuem. Isso ocorre especialmente às mulheres por sofrerem maior pressão psicológica para cuidar dos afazeres domésticos (Mattingly \& Sayer, 2006). Consequentemente, o tipo de atividade de lazer/tempo livre do gênero feminino depende da presença de filhos pequenos, bem como da estrutura de apoio que elas dispõem com empregados e/ou familiares (Jablonski, 2010). Portanto, não é possível identificar as 
diferentes funções que o lazer pode ter aos cientistas, ou seja, se serve como descanso, entretenimento, distração ou outras.

Em vista de aprofundamento dos resultados obtidos nesta pesquisa, novos estudos são sugeridos ultrapassando algumas das limitações da presente pesquisa. Por um lado, nosso estudo considerou uma centena de cientistas, sendo necessário mais estudos alargando o número de participantes de ambos os gêneros e abrangendo os diferentes campos das Ciências. Uma segunda limitação decorre da recolha da informação ter sido exclusivamente conduzida através de questionário estruturado de entrevista, muito assente no autorrelato dos participantes. Em futuros estudos seria interessante ter algum registo mais factual dos seus diários de trabalho ou ainda informação objetiva sobre seus projetos de pesquisa como volume de financiamento, pessoas participando nas equipes de pesquisa e publicações em revistas internacionais da respectiva especialidade tomando as diferentes áreas de pesquisa, pois a excelência não se assumirá em todas elas com as mesmas manifestações em termos de processos e de produtos. Desta forma, dados mais fidedignos com a realidade dos cientistas brasileiros podem ser conseguidos e confrontados com a informação de autorrelato, por forma a conseguirmos uma maior compreensão da excelência humana que, por ser um tema recente, apresenta ainda várias lacunas. Sobretudo é decisivo elencar, sendo possível, variáveis pessoais determinantes da excelência a que o processo educativo possa responder, assim como identificar os determinantes contextuais para que as instituições saibam adequar as suas dinâmicas e práticas ao desenvolvimento e concretização do talento humano.

\section{Referências}

Almeida, L. S., \& Wechsler, S. M. (2015). Excelência profissional: A convergência necessária de variáveis psicológicas. Estudos de Psicologia (Campinas), 32(4), $767-$ 775. doi: 10.1590/0103-166X2015000400019

Araújo, L. S., Cruz, J. F. A., \& Almeida, L. S. (2017). Achieving scientific excellence: An exploratory study of the role of emotional and motivational factors. High Ability Studies, 28(2), 249-264. doi: 10.1080/13598139.2016.1264293

Borsoi, I. C. F. (2012). Trabalho e produtivismo: saúde e modo de vida de docentes de instituições públicas de Ensino Superior. Cadernos de Psicologia Social do Trabalho, 15(1), 81-100. doi: 10.11606/issn.1981-0490.v15i1p81-100 
Borsoi, I. C. F., \& Pereira, F. S. (2013). Profesores do ensino público superior: Produtividades, produtivismo e adoecimento. Universitas Psychologica, 12(4), 1211. doi: 1233.10.11144/Javeriana.UPSY12-4.peps

Castro, M. T. L., \& Santo, W. R. S. (2017). Adoecimento do corpo docente na sociedade de vigilância e de controle. Arquivos em Movimento, 13(2), 86-101. Recuperado de https://revistas.ufrj.br/index.php/am/article/view/13502

Christensen, M., Dyrstad, J. M., \& Innstrand, S. T. (2018). Academic work engagement, resources and productivity: Empirical evidence with policy implications. Studies in Higher Education, 45(1), 1-14. doi: 10.1080/03075079.2018.1517304

Clement, J., (2008). Creative model construction in scientists and students: The role of imagery, analogy, and mental simulation. Dordrect: Springer.

Csikszentmihalyi, M. (1997). Creativity: Flow and the psychology of discovery and invention. New York: Harper Collins.

Damaske, S., Ecklund, E. H., Lincoln, A. E., \& White, V. J. (2014). Male scientists' competing devotions to work and family: Changing norms in a male-dominated profession. Work and Occupations, 41(4), 477-507. doi: 10.1177/0730888414539171

DeHaan, R. L. (2011). Teaching creative science thinking. Science, 334(6062), 1499-1500. doi: $10.1126 /$ science. 1207918

De Meis, L., Velloso, A., Lannes, D., Carmo, M. S., \& De Meis, C. (2003). The growing competition in Brazilian science: Rites of passage, stress and burnout. Brazilian Journal of Medical and Biological Research, 36(9), 1135-1141. doi: 10.1590/S0100879X2003000900001

Dubbelt, L., Rispens, S., \& Demerouti, E. (2016). Work engagement and research output among female and male scientists. Journal of Personnel Psychology. 15(2), 55-65. doi: $10.1027 / 1866-5888 / \mathrm{a} 000150$

Ecklund, E. H., \& Lincoln, A. E. (2016). Failing Families, Failing Science: Work-Family Conflict in Academic Science. New York: NYU Press.

Ericsson, K. A., Hoffman, R. R., Kozbelt, A., \& Williams, A. M. (Eds.). (2018). The Cambridge handbook of expertise and expert performance. United Kingtom: Cambridge University Press.

Fleith, D. S. (2007). Altas habilidades e desenvolvimento socioemocional. In D. S. Fleith \& E. M. L. S. Alencar (Eds.), Desenvolvimento de talentos e altas habilidades. Orientação a pais e professores (pp. 41-50). Porto Alegre: Artmed. 
Garcia-Santos, S. C., Almeida, L. S., \& Werlang, B. S. G. (2012). Human excellence: The contribution of personality. Paideia, 22(52), 271-279. doi: 10.1590/S0103$863 X 2012000200011$

Gómez, V. (2015). Moderación de la relación entre tensión laboral y malestar de profesores universitarios: Papel del conflicto y la facilitación entre el trabajo y la familia. Revista Colombiana de Psicologia, 24(1), 185-201. doi: 10.15446/rcp.v24n1.42081

González Ramos, A. M., \& Benavente, B. R. (2017). Excellence in science: A critical affirmative response. Cadernos de Pesquisa, 47(166), 1372-1394. doi: $10.1590 / 198053144233$

Grinnell, F. (2009). Everyday practice of science: Where intuition and passion meet objectivity and logic. Oxford, NY.: Oxford University Press.

Hadzigeorgiou, Y. (2016). Imaginative science education: The central role of imagination in science education. Switzer-land: Springer.

Hutz, C. S., \& Pacico, J. C., (2016). Psicologia Positiva: Avanços de uma nova abordagem. In A. P. Corrêa (Org.), Psicologia Positiva: Teoria e Prática (pp. 78-84). São Paulo: Leader.

Jablonski, B. (2010). A divisão de tarefas domésticas entre homens e mulheres no cotidiano do casamento. Psicologia: Ciência e Profissão, 30(2), 262-275. doi: 10.1590/S141498932010000200004

Lam, A. (2011). What motivates academic scientists to engage in research commercialization: 'Gold', 'ribbon' or 'puzzle'?. Research Policy, 40(10), 1354-1368. doi: $10.2139 / \mathrm{ssrn} .1815238$

Lemaitre, B. (2016). An essay on science and narcissism: How do high-ego personalities drive research in life sciences?. Lausanne: Autor.

Luthar, S. S. (2017). Doing for the greater good: What price, in academe?. Perspectives on Psychological Science, 12(6), 1153-1158. doi: 10.1177/1745691617727863

Mattingly, M. J., \& Sayer, L. C. (2006). Under pressure: Gender differences in the relationship between free time and feeling rushed. Journal of Marriage and Family, 68(1), 205-221. doi: 10.1111/j.1741-3737.2006.00242.x

Melo, H. P., Lastres, H. M. M., \& Marques, T. C. N. (2004). Gênero no sistema de ciências, tecnologia e inovação no Brasil. Revista Gênero, 4(2), 73-94. doi: 10.22409/rg.v4i2.247 
Montagner, M. I., \& Montagner, M. Â. (2010). Women and their careers at Unicamp's Faculty of Medical Sciences: Unique voices and collective images. História, Ciências, SaúdeManguinhos, 17(2), 379-397. doi: 10.1590/S0104-59702010000200007

Mundim, M. C. B., Wechsler, S. M., \& Silva Almeida, L. (2019). Dimensões Pessoais e Contextuais da Excelência: Estudo com Cientistas Brasileiros. Avaliaçao Psicologica, 18(4), 438-447. doi: 10.15689/ap.2019.1804.18578.12

Nielsen, M. W. (2016). Gender inequality and research performance: Moving beyond individual-meritocratic explanations of academic advancement. Studies in Higher Education, 41(11), 2044-2060. doi: 10.1080/03075079.2015.1007945

Pfeiffer, S. I. (2015). Tripartite model of giftedness and best practices in gifted assessment. Revista de Educacion, 368, 61-87. doi: 10.4438/1988-592X-RE-2015-368-293

Prado, R. M., \& Fleith, D. D. S. (2012). Pesquisadoras brasileiras: Conciliando talento, ciência e família. Arquivos Brasileiros de Psicologia, 64(2), 19-34. Recuperado de http://pepsic.bvsalud.org/scielo.php?script=sci_abstract\&pid=S1809$52672012000200003 \& \operatorname{lng}=$ pt\&nrm=iso

Retzbach, J., Otto, L., \& Maier, M. (2016). Measuring the perceived uncertainty of scientific evidence and its relationship to engagement with science. Public Understanding of Science, 25(6), 638-655. doi: 10.1177/0963662515575253

Ringelhan, S., Wollersheim, J., Welpe, I. M., Fiedler, M., \& Spörrle, M. (2013). Work motivation and job satisfaction as antecedents of research performance: Investigation of different mediation models. In A. Dilger, H. Dyckhoff, \& G. Fandel (Eds.), Performance Management im Hochschulbereich (pp. 7-38). Wiesbaden: Springer Gabler.

Seligman, M., \& Csikszentmihalyi, M. (2000). Positive Psychology: An introduction. American Psychologist, 55(1), 5-14. doi: 10.1037/0003-066X.55.1.5

Seligman, M. (2002). Positive psychology, positive prevention, and positive therapy. In C. R. Snyder, \& S. J. Lopez (Eds.), Handbook of positive psychology (pp. 3-9). New York: Oxford University Press.

Sewell, L., \& Barnett, A. G. (2019). The impact of caring for children on women's research output: A retrospective cohort study. PloS one, 14(3), e0214047. doi: 10.1371/journal.pone.0214047

Simonton, D. K. (1999). Talent and its development: An emergenic and epigenetic model. Psychological Review, 106(3), 435-457. doi: 10.1037/0033-295X.106.3.435 
Tahamtan, I., Afshar, A. S., \& Ahamdzadeh, K. (2016). Factors affecting number of citations: A comprehensive review of the literature. Scientometrics, 107(3), 1195-1225. doi: $10.1007 / \mathrm{s} 11192-016-1889-2$

Tang, C., \& Kaufman, J. C. (2017). Personal characteristics that distinguish creative scientists from less creative scientists. Journal of Creative Behavior, 51(3), 204-215. doi: 10.1002/jocb.99

Tauhed, S. Z., Rasdi, R. M., Ibrahim, R., \& Samah, B. A. (2019). The influence of networking, individual effort, and time management on research performance of academics at Malaysian research universities. Revista Publicando, 6(19), 325-338.

Trost, G. (2000). Prediction of excellence in school, higher education, and work. In K. Heller, F. Mönks, R. Sternberg, \& R. Subotnik (Orgs.), International Handbook of Giftedness and Talent (pp. 317-327). Oxford: Elsevier.

Van den Brink, M., \& Benschop, Y. (2012). Gender practices in the construction of academic excellence: Sheep with five legs. Organization, 19(4), 507-524. doi: $10.1177 / 1350508411414293$

Wai, J., Lubinski, D., \& Benbow, C. P. (2005). Creativity and occupational accomplishments among intellectually precocious youths: an age 13 to age 33 longitudinal study. Journal of Educational Psychology, 97(3), 484-492. doi: 10.1037/0022-0663.97.3.484

Zanotto, E. D. (2006). The scientists pyramid. Scientometrics, 69(1), 175-181. doi: 10.1007/s11192-006-0134-9

Zeng, A., Shen, Z., Zhou, J., Wu, J., Fan, Y., Wang, Y., \& Stanley, H. E. (2017). The science of science: From the perspective of complex systems. Physics Reports, 714, 1-73. doi: 10.1016/j.physrep.2017.10.001

Ziegler, A., \& Phillipson, S. N. (2012). Towards a systemic theory of gifted education. High Ability Studies, 23(1), 3-30. doi: 10.1080/13598139.2012.679085 


\section{Endereço para correspondência}

\section{Maria Célia Bruno Mundim}

Pontifícia Universidade Católica de Campinas

Campus II - Pós-graduação em Psicologia

Avenida John Boyd Dunlop, s/n, Jardim Ipaussurama, Campinas - SP, Brasil. CEP 13060-904

Endereço eletrônico: celiamundim@gmail.com

\section{Solange Muglia Wechsler}

Pontifícia Universidade Católica de Campinas

Campus II - Pós-graduação em Psicologia

Avenida John Boyd Dunlop, s/n, Jardim Ipaussurama, Campinas - SP, Brasil. CEP 13060-904

Endereço eletrônico: wechsler@lexxa.com.br

\section{Leandro Silva Almeida}

Universidade do Minho

Instituto de Educação, Campus de Gualtar, Braga, Portugal. CEP 4710-057

Endereço eletrônico: leandro@ie.uminho.pt

Recebido em: 13/09/2019

Reformulado em: 22/08/2020

Aceito em: 07/09/2020

\section{Notas}

* Psicóloga, doutora, com estágio pós-doutoral em Psicologia pela Pontifícia Universidade Católica de Campinas. Atualmente é pesquisadora colaboradora da mesma instituição.

** Professora Doutora do Programa de Pós-Graduação em Psicologia da Pontifícia Universidade Católica de Campinas.

*** Professor catedrático do Instituto de Educação / Universidade do Minho.

Este artigo de revista Estudos e Pesquisas em Psicologia é licenciado sob uma Licença Creative Commons Atribuição-Não Comercial 3.0 Não Adaptada. 